\title{
¿QUÉ TIPO DE GOBERNANZA PARA LA ECONOMÍA GLOBAL?'
}

\author{
Stefano ZAMAgni ${ }^{2}$
}

No hay duda de que el aumento endémico de las desigualdades sociales constituye en el actual tránsito de época uno de los nudos más inquietantes y difíciles de deshacer. Acerca de las desigualdades hoy sabemos casi todo: cómo se miden y con qué instrumentos; su tipología y dónde se encuentran con mayor frecuencia; cuáles son sus efectos sobre una gran variedad de frentes, tales como el económico, el político y el ético; cuáles son hoy los factores principalmente responsables, etc.

Sin embargo, no sabemos aún conceptualizarla. No conocemos su ontología y, por tanto, terminamos por asumirla como algo que es connatural a la condición humana o, por el contrario, como una especie de "mal necesario" para consentir ulteriores avances en nuestras sociedades. En general, la entendemos como algo con lo que es necesario aprender a convivir, así como en otras épocas de la historia la especie humana ha sabido hacer frente a las vicisitudes y los "caprichos" de la naturaleza. La aceptación supina del factum le corta las alas y la respiración al facendum. Y son, de hecho, muy escasas las propuestas creíbles que actualmente avanzan para hacer frente a este problema. El aumento de las desigualdades es, sobre todo, consecuencia del diseño institucional de la sociedad y de las reglas del juego económico que esta elige darse a sí misma.

Es, en vista de ello, que desde hace algún tiempo se está hablando, con una creciente insistencia, de la urgencia que hay de lograr una gobernanza global (la Caritas in veritate del papa Benedicto se ocupa en su última parte de este tema). Pero ¿qué modelo de gobernanza resulta hoy más eficaz? Para responder a esta pregunta, es necesario comprender la naturaleza específica de las causas que originan el fenómeno en cuestión. Comenzaré por aquellas de orden sociopolítico.

1 Publicado originalmente en Ferrara, P., Cheade, F., Zamagni, S., Cataldi, S. y Merletti, T. (2020). Governance. Roma: Città Nuova. Traducido con autorización del autor por Luis Fernando Ramírez, economista y magíster en gestión de empresas sociales para la innovación social y el desarrollo local. Actualmente funge como docente del Centro de Humanidades de la Universidad Pontificia Bolivariana. luisfernando.ramirez@upb.edu.co

2 Profesor de Economía en la Johns Hopkins University y la Università di Bologna. Actualmente es presidente de la Pontificia Academia de las Ciencias Sociales. 


\section{Capitalismo sin democracia}

Hasta hace poco tiempo el capitalismo estaba asociado de manera inquebrantable a la democracia. Si bien es cierto que ha habido épocas con una duración limitada en que esta sociedad entre capitalismo y democracia no ha estado presente, como en Corea del Sur o de Chile, así como lo sucedido en Europa durante la primera mitad del siglo XX, la gran novedad del mundo de hoy es que el vínculo entre democracia y capitalismo se ha rescindido. Se habla, de hecho, de orientalismo (Said, 1978) para significar que la civilización occidental ya no es más el modelo de referencia de la época global. El sueño de Francis Fukuyama de una democracia liberal global se ha roto miserablemente, mientras que el capitalismo ha triunfado en todo el mundo.

El hecho desconcertante, que muchos en Occidente parecen no haber entendido todavía, es que el nuevo capitalismo financiero (que ha avanzado más allá del capitalismo industrial) no tiene problemas para adaptarse a matrices religiosas, culturales y étnicas distintas. Las finanzas se han convertido en un fin en sí mismas, es decir, en autorreferenciales, y por tanto tienen una relación más remota y abstracta con el valor económico real al que debería estar conectada. De otro modo, las actividades especulativas del mercado financiero privan de cualquier tipo de estabilidad la relación entre el valor de los bienes y el modo en el que estos son representados en los distintos instrumentos financieros. Esto ha permitido, entre otras cosas, una expansión sin precedentes de la avidez y la irresponsabilidad. No había sucedido algo así con el "capitalismo nacional", que, por el contrario, se había erigido sobre los valores y sobre las tradiciones de la cultura occidental.

La novedad hoy es que se puede tener un capitalismo sin democracia y, en general, prescindiendo de los así llamados valores occidentales. En particular, el capitalismo "global" no necesita valerse del utilitarismo benthamiano ni del individualismo liberal para afirmarse. En la India, por ejemplo, se anteponen los vínculos comunitarios al éxito personal y se alimenta la identidad nacional para obstaculizar la invasión de los valores occidentales, aun así, este país, desde hace tiempo, se ha embarcado en la vía de la modernización capitalista. Podemos pensar también en los "valores asiáticos" propugnados por Lee Kuan Yew, el padre de la ciudad estado de Singapur. En China, las autoridades pueden llevar a prisión a quien juega abiertamente en la bolsa. Y así otros ejemplos.

Es, por tanto, equivocado pensar que la persistencia de tradiciones pre-modernas representa una forma de resistencia al capitalismo global. Al contrario, la fidelidad a esas tradiciones es aquello que permite a países como China, Singapur, la India, entre otros, recorrer el camino del proceso capitalista de modo, incluso, más radical que los países occidentales. Es fácil darse cuenta de esto: es más fácil remitirse a valores tradicionales para legitimar sacrificios e imposiciones de naturaleza antidemocrática a los propios ciudadanos.

En 1992, al respecto de la caída del Muro de Berlín, Francis Fukuyama publicó el famoso libro El fin de la historia. Destaca la tesis de este libro que el liberalismo y el capitalismo occidental han vencido finalmente su batalla contra el totalitarismo y los varios tradicionalismos. La superioridad intelectual de Occidente fue aplastante, y antes o después todo el mundo se habría adecuado a ella. 


\section{Populismo}

Pero la historia nos reserva sorpresas. Está aconteciendo, de hecho, que "nuestra" democracia liberal está cediendo espacio ante el populismo, concepción que ya no considera el pueblo llano como una categoría sociológica, sino como una categoría moral. La lucha política (para la ideología populista) es entre las virtudes (que le pertenecen al pueblo) y las no virtudes (que le pertenecen al pueblo), y el líder es aquel que logra encarnar el espíritu del pueblo. Por tal razón, el populismo rechaza la democracia representativa y opta a favor de la democracia directa. Pero aun las variantes democráticas del populismo no han tenido nunca éxito y rápidamente se han transformado en regímenes autoritarios. Podemos pensar en el caso reciente de Turquía, en que se están revalorizando los valores de las comunidades cerradas, aisladas y dirigidas por un hombre fuerte, o también los casos de Polonia, Hungría, Rusia, en que un autoritarismo blando se combina con el nacionalismo. Tómese nota de la siguiente asimetría: mientras que el mercado no tiene necesidad de la democracia para expandirse y crecer, la democracia liberal, por el contrario, necesita el mercado para realizarse plenamente.

¿Cómo ha podido suceder todo esto? El orden social del capitalismo occidental se encuentra privado de una dirección porque ha erosionado su fundamento. De hecho, la sociedad industrial ha tenido una base territorial nacional propia, lo cual no ha sucedido en la sociedad posindustrial en que el mercado es más grande que la soberanía y la necesidad de seguridad domina la necesidad de libertad. Después de haber tolerado e, incluso, favorecido, en décadas pasadas, la prevalencia de lo económico sobre lo político del mercado por encima de la democracia, nuestro mundo está hoy a la búsqueda de un sentido. Ni el Plattform-Kapitalismu, como lo llaman los alemanes, es decir, capitalismo de plataforma, es suficientemente robusto como para soportar un modelo de democracia como el liberal.

\section{Desvinculación moral}

Una de las causas de lo que está aconteciendo (ciertamente no es la única, pero tampoco es de las menores) es el afianzamiento de los lugares de la alta cultura de una desvinculación moral (moral disengagement), en el sentido de Albert Bandura (2015). Se trata de un caso grave del fracaso de la razón ampliamente promulgado en Occidente. En ética, se ha reflexionado ampliamente sobre la manera en que las personas logran comportarse moralmente, pero se nos ha olvidado "explicar" por qué las personas logran comportarse de un modo "deshumano" y conservar la autoestima y sentirse bien con su propia conciencia. Pensemos en el prominente movimiento de ideas alrededor de la ética en los negocios (business ethics) auspiciada por generosos, aunque no desinteresados, financiadores, y según la cual "lo que es bueno para el negocio es una buena ética" (good business is good ethic). La mayor parte de las empresas que afirman adherirse a los preceptos de la responsabilidad social corporativa (corporate social resposibility) publican anualmente el balance social, pero nunca se lee en tales documentos aquello que la empresa habría podido y, por tanto, debido hacer. Se describen solo las "bellas" iniciativas realizadas.

El aspecto inquietante de la desvinculación moral es que esta no altera los estándares morales socialmente aceptados, de este modo logra protegerse de las críticas sobre los principios morales, y sugiere, por el contrario, los medios para "eludir" aquellos estándares de un modo tal que parece proponer, más 
bien, los medios para neutralizar (azzerare) la responsabilidad de los agentes. Son ya bien conocidos los múltiples mecanismos gracias a los cuales los agentes logran este cometido: me autoconvenzo de que mi víctima es un ser subhumano o inferior para anular el remordimiento moral de haberla tratado de manera indigna. Es el desapego moral el que avala y nutre el así llamado relativismo moral, hoy rampante.

\section{Déficit cultural}

¿Qué encontramos en la base de una laguna tan grave? Un grave déficit cultural. En efecto, los textos de ética insisten y de manera casi exclusiva en la responsabilidad moral directa e indirecta del sujeto, pero nunca sobre la responsabilidad genérica (indistinta del punto de vista moral). Ya en la década de 1960, Pablo VI había aludido, en la perspectiva de la doctrina social católica, al concepto de estructuras de pecado, precisamente como ejemplo notable de responsabilidad genérica (la expresión "estructura de pecado" como tal fue acuñada por Juan Pablo II en la Sollicitudo rei socialis de 1987). Este es hoy el problema: puesto que la responsabilidad de los agentes es proporcional a su libertad, entonces se necesita entender de qué libertad gozan con respecto a las estructuras de mercado para evitar los daños que sus acciones provocan a aquellos que se encuentran distantes, pero que son afectados por los efectos de sus mecanismos causales.

Un libro reciente, de notable espesor cultural, es el de Katharina Pistor (2020). Para la abogada americana, es la ley (el "código del capital”), junto con el uso oportuno de esta, lo que está a la base de la acumulación y la conservación de la riqueza. La ley evoluciona y con frecuencia tiende a privilegiar ciertos tipos de capital y algunos de sus detentores con respecto a otros. La conclusión de la autora es que, si el capitalismo financiero y sus devastadoras desigualdades se han convertido en el problema de nuestro tiempo, no se lo debemos a las leyes naturales del mercado, sino a las leyes creadas por varios parlamentos que han escuchado más los cantos de las sirenas de los intereses de algunos pocos que los gemidos de los intereses de la multitud. Esta es la razón por la que es necesario intervenir globalmente para definir reglas jurídicas vinculantes para cada uno de los países.

\section{Macroeconomía}

¿Y qué podríamos decir de las causas de naturaleza propiamente económica responsable del aumento de la desigualdad de renta y de riqueza? Sabemos que son tres las razones principales por las que las desigualdades impactan negativamente la sostenibilidad económica de un país. En primer lugar, porque el aumento sistemático de las desigualdades refleja la presencia dentro del sistema de rentas económicas que derivan de actividades de tipo extractivo, y como se sabe al menos desde los tiempos de David Ricardo (a inicios de 1800), la renta es improductiva y, por tanto, limita el proceso de crecimiento. En segundo lugar, la desigualdad favorece la práctica de aquellas formas de economía denominadas "plutonomía" (la economía para los superricos): pequeños grupos de población que alimentan modelos neoconsumistas, que se manifiestan en el derroche de distintos recursos, sobre todo, ambientales, por no hablar de las consecuencias de naturaleza social y cultural. Por último, el crecimiento de la desigualdad es un indicador confiable de inestabilidad financiera, con las consecuencias que hoy lamentablemente conocemos bien. 
¿Por qué es importante hacer memoria de lo descrito? Porque la tendencia prevalente en el debate público sobre el tema es atribuir las causas de la desigualdad a múltiples problemas de funcionamiento de los mercados locales, en especial los del mercado del trabajo y su conocido desequilibrio entre oferta y demanda. En este caso, las recomendaciones son bien conocidas: reforzar la posición en el mercado del agente individual, trabajador o emprendedor que este sea, interviniendo en el sistema educativo, en el sistema de salud de las personas, en la información, en la administración de las empresas, etc.

\section{Finanzas globales}

Aun sin negar la relevancia de estas consideraciones, es necesario comenzar a hablar abiertamente del hecho de que son, por el contrario, las fuerzas de tipo macroeconómico (aquellas que definen la estructura del sistema) las primeras responsables del aumento de las desigualdades. En particular, son las características asumidas por las finanzas globales de manera sistemática en el curso de los últimos cuarenta años la causa principal del fenómeno en discusión. Como lo explica con lucidez Maureen O'Hara (2016), la novedad de las finanzas modernas es su autorreferencialidad: las finanzas se han convertido en fines para sí mismas. Esto ha sucedido porque las finanzas modernas no se ocupan de forma prevalente de títulos financieros, como acciones, obligaciones, hipotecas, etc., sino de flujos de caja, es decir, del dinero derivado de los pagos de intereses, utilidades, etc. Tales flujos se combinan entre ellos para crear una especie de "productos" financieros. Es interesante anotar que también la Organisation for Economic Co-operation and Development (OECD) así lo ha reconocido cuando escribe en su informe In it together: Why less inequality benefits all (2015) que el crecimiento de las desigualdades es parte integral de las actuales estructuras económicas. Y, por tanto, si en realidad queremos afrontar el fenómeno, es necesario intervenir internacionalmente con acuerdos focalizados, como ahora se busca hacer con el problema ambiental.

\section{Anomalías}

Un cuidadoso estudio reciente, "Welfare State, Inequality, and Globalization: Role of Internationalcapital-flow Direction" (Razin y Sadka, 2019), nos ofrece una confirmación de peso en relación con lo que hemos afirmado. A partir de la consideración de que la globalización financiera, es decir, en ausencia de intervenciones adecuadas por parte de la autoridad, crea típicamente ganadores y perdedores, los autores documentan cómo las consecuencias que se producen o se derivan de las desigualdades de renta dependen del hecho de si un país es exportador o importador de capitales en relación con el resto del mundo. En el primer caso, la globalización financiera impulsa hacia lo alto los rendimientos sobre los ahorros y reduce los salarios; en el segundo, sucederá lo contrario. Por tanto, técnicamente es posible intervenir para hacer de modo que los ahorros derivados por la libre circulación de capitales y el trabajo se distribuyan de manera equitativa entre los distintos grupos sociales. Pero para ello se necesita voluntad, algo que aún no sucede, salvo en casos rarísimos. Ha llegado el momento de denunciar con fuerza la creencia errónea, conducida por el pensamiento neoliberal, según la cual no habría nada que hacer para corregir las anomalías de la globalización financiera sobre la desigualdad. 
No es una buena ciencia aquella que invoca razones de naturaleza técnica para ocultar elecciones precisas de naturaleza ideológica. Ha escrito Aristóteles que el discurso sobre la igualdad y la desigualdad se vierte sobre criterios de distribución de bienes y recursos entre grupos, y trata de individualizar los modos de tratar de manera adecuada la diversidad entre ciudadanos. Es, por tanto, incorrecto (por no decir mistificador) intentar legitimar, e incluso justificar, la desigualdad como medida para no renunciar a los incentivos del trabajo, para premiar el mérito y, en definitiva, para asegurar la eficiencia. Porque la eficiencia (como escribiría Vilfredo Pareto, inventor del concepto a finales de 1800) pertenece al orden de los medios y no aquel de los fines. Por tal razón, es necesario definir "antes que nada" cuál es el fin por el que se pretende ser eficientes y eso exige que se declare en el juicio de valor que se elige adoptar. Existe una responsabilidad precisa que recae en cabeza de los profesionales de la economía, quienes, aun siendo conscientes de los efectos redistributivos de la globalización hasta hace poco tiempo, no habían hablado o discutido sobre ello fuera de los círculos académicos. Esto quizá por temor de ofrecerles argumentos a aquellos demagogos soberanistas; pero el resultado finalmente se ha visto: la hegemonía del pensamiento único ha terminado por alimentar a los extremistas más desesperados (Zamagni, 2019).

\section{¿Cuánto gana el administrador delegado (CEO pay ratio)?}

Un modo simple, aunque a veces apasionado, para medir la desigualdad es determinar cuánto aumentaría la renta media de un individuo porcentualmente si la renta general fuese distribuida de manera igualitaria entre todos. Un indicador que es mencionado cada vez con mayor frecuencia para ilustrar el aumento de la desigualdad es el $C E O$ pay ratio: la relación que indica cuántas veces la remuneración del administrador delegado (chief executive officer [CEO]) supera la remuneración media de sus empleados. Según Pearl Meyer, ${ }^{3}$ en 2018, en los Estados Unidos, esta relación alcanzó en promedio 144 veces, un dato que sube a 337 veces en las sociedades con más de 10000 empleados, y en una docena de casos esa relación ha alcanzado 1000 veces. Incluso, quien no es un economista puede comprender que las cifras de este tipo no tienen ninguna justificación de naturaleza económica.

La bien conocida escuela de pensamiento neoclásica elaboró, desde los tiempos de la revolución marginalista a finales de 1800, la bien conocida teoría de la distribución de las rentas según la productividad marginal: cada factor de producción debería recibir una remuneración proporcional a su productividad, es decir, según su contribución en la obtención del producto final. Pero ¿puede pensarse que existe en el mundo un sujeto cuya productividad marginal es de 1000 o incluso 337 veces superior a aquella del promedio de la empresa que administra? Perplejidades de este tipo comienzan a abrirse espacio en la opinión pública. Desde 2018, las sociedades americanas listadas en bolsa deben indicar, como una regla de transparencia, su CEO pay ratio (el Reino Unido introducirá esta misma medida a partir de 2020). El fin que se proponen tales procedimientos es llegar a contener, dentro de los límites de la decencia civil más que ética, los excesos de la autorreferencialidad del management.

3 Sociedad americana especializada en el monitoreo de las compensaciones para ejecutivos (https://www.pearlmeyer.com/). 


\section{Acaparar la tierra (land grabbing)}

Quiero referirme, a continuación, a otra causa que agrava las desigualdades en tiempos recientes. Me refiero al fenómeno del acaparamiento de tierras (land grabbing); es cierto que no se trata de algo nuevo, pero que sí ha crecido en intensidad en la medida en que avanza la reducción de suelos disponibles por cuenta del cambio climático y que, como se sabe, impacta negativamente la fertilidad de las tierras. La carrera por el acaparamiento de las tierras es su triste consecuencia. Desde 2000 hasta hoy, han sido sustraídas a las comunidades locales que la cultivan cerca de 88 millones de hectáreas de tierras fértiles. Como es sabido, el instrumento jurídico adoptado (contratos de venta o de arriendo a largo plazo establecidos con los campesinos) ofrece una fachada de legitimidad que no satisfacen de facto el requisito de licitud. Es tanto así que la Organización de las Naciones Unidas (ONU) ha (finalmente) lanzado la iniciativa UN Treaty para dictar reglas que garanticen el respeto de los derechos humanos, tales como el acceso y la posesión de la tierra.

Como se observa en la base de datos Land Matrix, que desde 2012 monitorea el fenómeno con un trabajo de atenta lectura de los contratos, los mayores acaparadores son los Estados Unidos, el Reino Unido, los Países Bajos, pero también la India y Brasil. Italia, por su parte, ha acaparado algo más de un millón de hectáreas en países africanos como Gabón, Liberia, Etiopía, Senegal e, incluso, Rumania. Una mirada rápida permite observar que las poblaciones expulsadas de sus tierras luego irán a engrosar los flujos migratorios y alimentar las injusticias de las que los diarios puntualmente nos informan (Franzini y Raitano, 2018).

\section{Propuestas}

¿Cómo razonar sobre los mecanismos generadores de desigualdad? La urgencia de intervenir sobre los mecanismos de funcionamiento del mercado global para reducir las desigualdades ha sido la preocupación principal del trabajo científico de Anthony Atkinson, el famoso economista de Oxford quien murió recientemente. En su último libro Inequality: What can be done? (2015), Atkinson plantea quince propuestas concretas para afrontar el problema, sobre las cuales se puede ciertamente discrepar, pero de las cuales no se puede prescindir.

Distinguiendo entre políticas pre-distributivas (aquellas que actúan sobre las dotaciones y las oportunidades de vida de los individuos) y aquellas redistributivas (que modifican las reglas del juego prevalente), Atkinson sugiere modificar en sentido progresivo los sistemas fiscales, para combatir la evasión fiscal mediante el cierre de los paraísos fiscales, de realizar planes puntuales de inversión sobre el capital humano (y no genéricos), de adoptar un salario mínimo de decencia humana, de insertar una cláusula específica de naturaleza distributiva en la legislación con respecto a las reglas de la competencia, de intervenir en materia hereditaria para corregir la transmisión intergeneracional de las desigualdades, entre otros. 


\section{Estructuras impositivas}

Una propuesta reciente, que se discute bastante, es aquella planteada por los norteamericanos Eric Posner y Glen Weyl en Radical markets: Uprooting capitalism and democracy for a just society (2018). Su punto de partida es la toma de conciencia sobre el hecho de que el aumento de las desigualdades representa hoy la amenaza más seria a la sostenibilidad económica del mercado capitalista. Este aumento no es el precio que se debe pagar para garantizar una economía dinámica, como lo proponía el pensamiento neoliberal. Una prueba de ello es que en el curso de los últimos treinta años se observa un fenómeno nunca antes visto: la stagnequality, esto es, un crecimiento más bajo (y más incierto) que se acompaña de una desigualdad más alta. Este es un fenómeno completamente simétrico a aquel de la estanflación (stagnation) de las década de 1960 y 1980, cuando el estancamiento de la economía se asoció con un aumento de la inflación. Fue con la Administración Reagan en los Estados Unidos y con la de Thatcher en Inglaterra que el programa neoliberal tomó vuelo. Hoy sabemos que la promesa de tolerar un poco de desigualdad para obtener mayor crecimiento no solo no puede ser mantenida, sino que además agrava la perspectiva de crecimiento.

Sin embargo, la propuesta, verdaderamente original, de Posner y Weyl, es aquella de trasladar la estructura de impuestos del trabajo y de las actividades productivas a las varias formas de propiedad, inmobiliaria, financiera o cualquier otra. Todo ciudadano es libre de fijar el valor de aquello que posee, bajo la condición de aceptar vender (o arrendar) su bien patrimonial si le llegase una oferta superior a la valoración por él indicada. De esta manera, el ciudadano no tendría ningún incentivo para declarar valores demasiado bajos, pero tampoco valores exageradamente altos para no tener que pagar luego impuestos muy elevados. En buena sustancia, en la base de la propuesta se encentra el intento de activar una evolución del capitalismo a partir de una gradual transformación de los derechos de propiedad, visto como una antesala del monopolio.

\section{Concentración de riqueza}

Sin duda, Adam Smith en su libro La riqueza de las naciones (1776) había observado que el modo más indoloro para que una empresa incremente sus utilidades no era tanto aquel de innovar y de esforzarse por reducir los costos de producción, sino más bien alcanzar acuerdos anticompetitivos con otras empresas, y de esta manera incrementar su propio poder de mercado. Por esta razón, desde finales de 1800, se introducen las leyes antimonopolio (antitrust), la primera de las cuales fue la célebre Sherman Act de 1891 en los Estados Unidos. Con la llegada de la globalización, la capacidad de control efectivo de las distintas autoridades se considera que ha disminuido (como todos lo saben) y hoy las empresas de la alta tecnología (bigh tech) han encontrado otras formas para lucrarse con utilidades monopolísticas, modos no rastreables por los organismos de vigilancia. Hoy, estudiosos como Joseph Stiglitz, Robert Reich, Dani Rodrik, así como tanques de pensamiento (think tank) como el Boston Consulting Group, el Anderson Institute, L'Economist, Financial Times se han unido para poner en luz la peligrosa concentración de riqueza en manos de oligopolistas, frecuentemente sin escrúpulos éticos. El peligro no es solo de naturaleza económica, sino que concierne también al destino de la democracia, dado que quien controla los medios termina también (como recodaba von Hayek) determinando los fines. Es la historia la que nos enseña que la hegemonía económica se traduce, antes o después, en hegemonía cultural y política. 
En su monumental ensayo reciente Capital and ideology (2020), Thomas Piketty lanza propuestas igualmente radicales en que sugiere intervenir, en especial, en el capital y la propiedad de los recursos naturales, más que en el conocimiento. El ejemplo que toma en consideración el economista francés es el de Rusia. En un país donde estaba prohibida la propiedad privada, todos los recursos naturales están ahora bajo el control de diez oligarcas. En este país, no existe impuesto alguno a la sucesión, mientras que sobre la renta existe una tasa impositiva fija (flat tax) del $13 \%$ para todos. También en China no existe un impuesto a las sucesiones, mientras que en Corea del Sur y en Taiwán la misma tasa alcanza el $50 \%$. En el Reino Unido, es gracias a un impuesto a las sucesiones del $80 \%$ sobre el patrimonio que, desde la primera época de posguerra en adelante, se ha experimentado una redistribución de la propiedad de la tierra.

\section{Gobernanza global}

Todo lo que hemos escrito hasta ahora sugiere que, si se quiere comprender el indigno fenómeno de la injusticia social, es necesario pensar seriamente en un modelo creíble de gobernanza global. ¿Cuál es la dificultad? Conciliar las reglas de la gobernanza interna de los países (cada uno de los cuales tiene su propia historia específica, sus normas sociales de comportamiento, su matriz cultural) con la uniformidad de las reglas que inevitablemente caracterizan la gobernanza global.

Pero no puede olvidarse nunca que, de hecho, los vínculos externos al país cuando esto debe dar forma a las propias políticas nacionales implican un costo respecto de la legitimidad democrática (costo que, como en este tiempo acontece, termina con el refuerzo de las iniciativas hacia el populismo soberanista). Se trata, entonces, de elegir entre dos concesiones alternativas de gobernanza económica global, que el economista norteamericano Dani Rodrik, en "Putting global governance in its place" (Rodrik, 2020), ha denominado globalization enhacing global governance (gobierno mundial que favorece la globalización) y democracy-enhancing global governance (gobierno global que favorece el desarrollo de la democracia).

La idea de fondo de la segunda opción es que, cuando se mete mano al diseño de las reglas transnacionales, es necesario insertar entre los objetivos a perseguir no solo el aumento de la eficiencia en la colocación de los recursos, sino también la ampliación de las bases democráticas. Para decirlo de otra forma, si bien es cierto que la globalización aumenta el espacio de los derechos humanos negativos (es decir, la libertad para), restringe el espacio, si no se corrige con cláusulas de salvaguardia social de los derechos humanos positivos (es decir, la libertad de). Es, por tanto, necesario activar un auténtico proyecto de transformación para el diseño actual del orden económico global.

\section{Referencias}

Atkinson, A. B. (2015). Inequality: What Can Be Done? Cambridge: Harvard University Press.

Bandura, A. (2015). Moral disengagement: How people do harm and live with themselves. Nueva York: Macmillan Learning.

Ferrara, P., Cheade, F., Zamagni, S., Cataldi, S. y Merletti, T. (2020). Governance. Roma: Città Nuova. 
Franzini, M. y Raitano, M. (eds.) (2018). Il mercato rende diseguali? La distribuzione dei redditi in Italia. Bolonia: Il Mulino.

Fukuyama, F. (1992). El fin de la historia y el último hombre. Planeta.

O'Hara, M. (2016). Something for nothing: Arbitrage and ethics on wall street. Nueva York: WW Norton \& Company.

Organisation for Economic Co-operation and Development. (2015). In it together: Why less inequality benefits all. https://www.oecd.org/social/in-it-together-why-less-inequality-benefits-all-9789264235120-en.htm

Piketty, T. (2020). Capital and ideology. Cambridge: Harvard University Press.

Pistor, K. (2020). The code of capital: How the law creates wealth and inequality. Princeton: Princeton University Press. https://doi.org/10.1515/9780691189437

Posner, E. A. y Weyl, E. G. (2018). Radical markets: Uprooting capitalism and democracy for a just society. Princeton: Princeton University Press.

Razin, A. y Sadka, E. (2019). Welfare state, inequality, and globalization: Role of international-capital-flow direction. Working Paper, 25772. DOI 10.3386/w25772

Rodrik, D. (2020). Putting global governance in its place. The World Bank Research Observer, 35(1), 1-18. https:// doi.org/10.1093/wbro/lkz008

Said, E. W. (1978). Orientalism. Nueva York: Pantheon Books.

Smith, A. (2020). La riqueza de las naciones (Décima rei). Alianza Editorial.

Zamagni, S. (2019). Responsabili: come civilizzare il mercato. Bolonia: Il Mulino. 